(MRF9200006). The authors state that there are no conflicts of interest to disclose.

Ella M. Meumann ${ }^{1,2,3}$, Dimitrios Menouhos ${ }^{1}$, Stefanos Christofis ${ }^{1}$, Kwaku M. Kondambu-Saaka ${ }^{1}$, Jaimee Harbidge $^{1}$, Farshid Dakh ${ }^{1}$, Kevin Freeman ${ }^{1}$, Robert Baird ${ }^{1,2}$

${ }^{1}$ Territory Pathology, Royal Darwin Hospital, Darwin, Australia; ${ }^{2}$ Department of Infectious Diseases, Division of Medicine, Royal Darwin Hospital, Darwin, Australia; ${ }^{3}$ Global and Tropical Health Division, Menzies School of Health Research and Charles Darwin University, Darwin, Australia

Contact Dr Ella Meumann.

E-mail: ella.meumann@menzies.edu.au

1. Wu F, Zhao S, Yu B, et al. A new coronavirus associated with human respiratory disease in China. Nature 2020; 579: 265-9.

2. Andersson P, Sherry NL, Howden BP. Surveillance for SARS-CoV-2 variants of concern in the Australian context. Med J Aust 2021; 214 500-502.e1.

3. Lane CR, Sherry NL, Porter AF, et al. Genomics-informed responses in the elimination of COVID-19 in Victoria, Australia: an observational, genomic epidemiological study. Lancet Public Health 2021; 6: e547-56.

4. Rockett RJ, Arnott A, Lam C, et al. Revealing COVID-19 transmission in Australia by SARS-CoV-2 genome sequencing and agent-based modeling. Nat Med 2020; 26: 1398-404.

5. Hoang T, da Silva AG, Jennison AV, et al. AusTrakka: fast-tracking nationalized genomics surveillance in response to the COVID-19 pandemic. Nat Commun 2022; 13: 865 .

6. Campbell F, Archer B, Laurenson-Schafer $\mathrm{H}$, et al. Increased transmissibility and global spread of SARS-CoV-2 variants of concern as at June 2021. Euro Surveill 2021; 26.

7. Network for Genomic Surveillance in South Africa. Briefing on COVID-19 and vaccination programme developments 25. Cited 12 Dec 2021. https://www.youtube.com/watch?v=4vIxzO1iVo0.

8. Viana R, Moyo S, Amoako DG, et al. Rapid epidemic expansion of the SARS-CoV-2 Omicron variant in southern Africa. Nature 2022; 603: 679-86.

9. The Northern Territory Government. Northern Territory economy: population. Cited 10 Jun 2021. https://nteconomy.nt.gov.au/population

10. O'Toole A, Scher E, Underwood A, et al. Assignment of epidemiological lineages in an emerging pandemic using the pangolin tool. Virus Evol 2021; 7: veab064.

11. Katoh K, Misawa K, Kuma K, et al. MAFFT: a novel method for rapid multiple sequence alignment based on fast Fourier transform. Nucleic Acids Res 2002; 30: 3059-66.

12. Nguyen LT, Schmidt HA, von Haeseler A, et al. IQ-TREE: a fast and effective stochastic algorithm for estimating maximum-likelihood phylogenies. Mol Biol Evol 2015; 32: 268-74.

DOI: https://doi.org/10.1016/j.pathol.2022.03.005

\section{Sensititre broth microdilution plates variation in colistin quality control minimum inhibitory concentration results}

To the Editor,

Multi-drug-resistant (MDR) Gram-negative organisms represent a significant healthcare problem. Many are associated with hospital-acquired infections, with Pseudomonas aeruginosa being one of the most commonly identified pathogens. ${ }^{1}$ Carbapenem resistance significantly limits available treatment options with polymyxins typically being some of the last-line drugs available. ${ }^{2}$ The current recommended method for susceptibility testing of colistin is broth microdilution. ${ }^{3,4}$ It is also recommended that concurrent quality control testing should be performed with a drugsusceptible strain (Escherichia coli ATCC 25922 or Pseudomonas aeruginosa ATCC 27853), and a drug-resistant strain (Escherichia coli NCTC 13846). ${ }^{5}$ According to current European Committee on Antimicrobial Susceptibility Testing (EUCAST) guidelines, the target minimum inhibitory concentration (MIC) (and range) are as follows: E. coli ATCC $25922,0.5-1 \mathrm{mg} / \mathrm{L}(0.25-2 \mathrm{mg} / \mathrm{L}) ; P$. aeruginosa ATCC 27853, 1-2 mg/L (0.5-4 mg/L); E. coli NCTC 13846, $4 \mathrm{mg} / \mathrm{L}(2-8 \mathrm{mg} / \mathrm{L}) .{ }^{5}$ Clinical and Laboratory Standards Institute (CLSI) expected MIC ranges for E. coli ATCC 25922 and $P$. aeruginosa ATCC 27853 are the same as for EUCAST but do not specify target MICs. 5,6

We performed susceptibility testing for colistin and polymyxin B using a commercial broth microdilution method (Sensititre; Thermo Fisher, USA) following an internal evaluation in $2017 .^{4}$ Although polymyxin B was included in the plates, testing with colistin is generally recommended, and there are currently no breakpoints available for polymyxin B. The colistin MIC results were interpreted based on EUCAST breakpoints. ${ }^{3}$ Testing was usually performed for carbapenem-resistant organisms or on request by clinicians. Since the start of testing, three different plates were in use (sequentially) for colistin and polymyxin B testing: GNX3F, SGP1MBD and SGPMDRO1F. These plates are subsequently referred to as Plates $\mathrm{A}, \mathrm{B}$, and $\mathrm{C}$, respectively. Plate $\mathrm{A}$ was a research use only plate, and Plates $B$ and $C$ were customised in vitro diagnostic plates. All three plates contained colistin and polymyxin $\mathrm{B}$, with wells ranging from $0.25 \mathrm{mg} / \mathrm{L}$ to $4 \mathrm{mg} / \mathrm{L}$.

Quality control data and colistin/polymyxin B testing results between September 2017 and May 2021 were collated. Enterobacterales and $P$. aeruginosa (as identified by MALDI Biotyper; Bruker, USA) were included. These data are summarised in Fig. 1. The quality control results demonstrated a rightward shift (increased MIC) in the quality control results of $E$. coli ATCC 25922 during the period when Plate B was in use compared to Plates A and C. Quality control testing with $P$. aeruginosa ATCC 27853 was not performed when Plate A was in use, but a leftward shift was demonstrated between using Plates B and C. Conversely, the majority of the mcr-1 positive E. coli NCTC 13846 had MIC $>4 \mathrm{mg} / \mathrm{L}$, which is outside of the target MIC of $4 \mathrm{mg} / \mathrm{L}$.

Although within the expected range, many of the quality control (QC) results were outside of the target MICs for $P$. aeruginosa ATCC 27853 when Plate B was in use. A similar rightward shift was also seen in the MIC distribution of clinical isolates during this period. Colistin resistance rates for the tested Enterobacterales stratified by testing plates were: Plate A, $11.8 \%$ (4/34); Plate B, 6.5\% (5/77); and Plate C, $15.0 \%$ (3/20). Colistin resistance rates for $P$. aeruginosa were: Plate A, 5.5\% (3/55); Plate B, 46.2\% (54/117); and Plate C, 2.6\% (1/38). There was a clear increase in the number of $P$. aeruginosa with resistant MICs when Plate B was in use, which was reversed when we switched to Plate C. The most commonly tested Enterobacterales were Escherichia coli and Klebsiella pneumoniae. The MIC distributions 


\begin{tabular}{|c|c|c|c|c|c|c|c|c|c|c|c|c|}
\hline \multirow{2}{*}{$\begin{array}{c}\text { Sensititre } \\
\text { plate }\end{array}$} & \multicolumn{6}{|c|}{ Colistin (MIC in mg/L) } & \multicolumn{6}{|c|}{ Polymyxin B (MIC in mg/L) } \\
\hline & 0.25 & 0.5 & 1 & 2 & 4 & $>4$ & 0.25 & 0.5 & 1 & 2 & 4 & $>4$ \\
\hline \multicolumn{13}{|c|}{ Escherichia coli ATCC 25922} \\
\hline A & 37 & 2 & 0 & 0 & 0 & 0 & 37 & 2 & 0 & 0 & 0 & 0 \\
\hline $\mathrm{B}$ & 0 & 8 & 47 & 4 & 0 & 0 & 1 & 0 & 39 & 19 & 0 & 0 \\
\hline $\mathrm{C}$ & 22 & 0 & 0 & 0 & 0 & 0 & 21 & 1 & 0 & 0 & 0 & 0 \\
\hline \multicolumn{13}{|c|}{ Escherichia coli NCTC 13846} \\
\hline$A$ & 0 & 0 & 0 & 0 & 0 & 25 & 0 & 0 & 0 & 0 & 0 & 25 \\
\hline $\mathrm{B}$ & 0 & 0 & 0 & 0 & 17 & 12 & 0 & 0 & 0 & 3 & 22 & 4 \\
\hline C & 0 & 0 & 0 & 0 & 0 & 3 & 0 & 0 & 0 & 0 & 0 & 3 \\
\hline \multicolumn{13}{|c|}{ Pseudomonas aeruginosa ATCC 27853} \\
\hline A & \multicolumn{12}{|c|}{ N/A } \\
\hline $\mathrm{B}$ & 0 & 0 & 0 & 17 & 12 & 0 & 0 & 0 & 0 & 22 & 7 & 0 \\
\hline C & 0 & 0 & 20 & 0 & 0 & 0 & 0 & 0 & 20 & 0 & 0 & 0 \\
\hline \multicolumn{13}{|c|}{ Clinical Pseudomonas aeruginosa isolates } \\
\hline A & 2 & 2 & 37 & 11 & 1 & 2 & 0 & 3 & 48 & 5 & 1 & 1 \\
\hline $\mathrm{B}$ & 0 & 0 & 12 & 51 & 51 & 3 & 0 & 0 & 10 & 64 & 47 & 1 \\
\hline $\mathrm{C}$ & 0 & 1 & 20 & 16 & 0 & 1 & 0 & 0 & 30 & 7 & 0 & 1 \\
\hline \multicolumn{13}{|c|}{ Clinical Escherichia coli isolates } \\
\hline A & 8 & 0 & 0 & 0 & 0 & 0 & 7 & 1 & 0 & 0 & 0 & 0 \\
\hline $\mathrm{B}$ & 1 & 2 & 18 & 0 & 0 & 2 & 1 & 0 & 9 & 11 & 0 & 1 \\
\hline $\mathrm{C}$ & 5 & 0 & 0 & 0 & 0 & 0 & 4 & 1 & 0 & 0 & 0 & 0 \\
\hline \multicolumn{13}{|c|}{ Clinical Klebsiella pneumoniae isolates } \\
\hline A & 19 & 0 & 1 & 0 & 0 & 3 & 17 & 2 & 1 & 0 & 0 & 3 \\
\hline$B$ & 3 & 0 & 29 & 7 & 1 & 2 & 1 & 3 & 2 & 34 & 1 & 1 \\
\hline$C$ & 7 & 1 & 1 & 0 & 0 & 2 & 7 & 2 & 0 & 0 & 0 & 2 \\
\hline
\end{tabular}

Fig. 1 Colistin and polymyxin B minimum inhibitory concentration distributions of quality control strains and clinical isolates stratified by Sensititre plates. Green shading indicates target MIC for quality control strain, and orange shading indicates the accepted range.

are shown in Fig. 1, and demonstrated a similar rightward shift among susceptible isolates when Plate B was in use, which was also reversed when we switched to Plate C.

Pseudomonas aeruginosa naturally have higher MICs compared to Enterobacterales. Wild-type isolates (including $P$. aeruginosa 27853) may be expected to have MICs of up to $4 \mathrm{mg} / \mathrm{L}$, which would be interpreted as resistant, based on current breakpoints (susceptible $\leq 2$, resistant $>4$ ), although within the area of technical uncertainty. Due to typical MICs being close to the breakpoint, minor shifts in MIC results in a categorical change of interpretation from susceptible to resistant.

Although broth microdilution is recommended as the test method for colistin, there are potential limitations. It is not practical for diagnostic laboratories to routinely perform broth microdilution and there is still reliance on commercial testing methods such as Sensititre that has been previously validated by EUCAST. Despite previous validation data, variations may occur between different plates, which emphasise the need for adequate quality control testing to monitor the performance of these tests. Shifting MIC ranges of QC strains can result in major errors, particularly for organisms such as $P$. aeruginosa with typical MICs close to the breakpoint.

While the QC results were largely within range, many occurred outside of the target MICs for both colistinsusceptible and colistin-resistant QC isolates. Inclusion of colistin-susceptible and colistin-resistant QC strains is important to ensure reliable results across the tested MIC range. Some of the expected MIC ranges of quality control strains occur at the lower and upper limits of range of tested MICs Manufacturers may consider increasing the tested MIC range as an additional measure to improve robustness of their tests. Diagnostic laboratories should be aware of testing limitations and importance of closely monitoring quality control results, particularly if quality control results are skewed towards one end of the expected range outside of target range.

Conflicts of interest and sources of funding: The authors state that there are no conflicts of interest to disclose.

Ka Lip Chew, Clayton Low, Rosemini Achik, Roland Jureen, Kean Lee Chew

Department of Laboratory Medicine, National University Hospital, Singapore

Contact Dr Ka Lip Chew.

E-mail: ka_lip_chew@nuhs.edu.sg

1. Cai Y, Venkatachalam I, Tee NW, et al. Prevalence of healthcareassociated infections and antimicrobial use among adult inpatients in Singapore acute-care hospitals: results from the first national point prevalence survey. Clin Infect Dis 2017; 64: S61-7.

2. Chew KL, Octavia S, Ng OT, et al. Challenge of drug resistance in Pseudomonas aeruginosa: clonal spread of NDM-1-positive ST-308 within a tertiary hospital. J Antimicrob Chemother 2019; 74: 2220-4.

3. The European Committee on Antimicrobial Susceptibility Testing. Breakpoint tables for interpretation of MICs and zone diameters. Version 11.0. 2021. http://www.eucast.org

4. Chew KL, La M-V, Lin RTP, Teo JWP. Colistin and polymyxin B susceptibility testing for carbapenem-resistant and mcr-positive 
Enterobacteriaceae: comparison of Sensititre, Microscan, Vitek 2, and Etest with broth microdilution. J Clin Microbiol 2017; 55: 2609-16.

5. The European Committee on Antimicrobial Susceptibility Testing. Routine and extended internal quality control for MIC determination and disk diffusion as recommended by EUCAST. Version 11.0. 2021. http:// www.eucast.org

6. Clinical and Laboratory Standards Institute (CLSI). Performance Standards for Antimicrobial Susceptibility Testing. CLSI supplement M100. 30th ed Wayne, PA: Clinical and Laboratory Standards Institute, 2020.

DOI: https://doi.org/10.1016/j.pathol.2021.08.016

\section{First human case of infection with Arsenophonus nasoniae, the male killer insect pathogen}

\section{To the Editor,}

Many arthropod-borne human infections have been recognised in recent years, but this is the first reported case of honeybee-associated Arsenophonus nasoniae bacteraemia.

We present the case of a 37-year-old male who attended the hospital emergency department with sudden onset of fever, rigors, and myalgia 5 hours after sustaining a bee sting to his left thumb that occurred in the evening during April 2018 behind his home garage in Wollongong, New South Wales, Australia. This reaction was unusual for him as he had sustained several bee stings in the past without any severe effects. His other medical problems included eczema, well-controlled with topical corticosteroid cream, and chronic neck pain.

On presentation, he was well-perfused with blood pressure of $137 / 81 \mathrm{mmHg}$ but was tachypnoeic with a respiratory rate of 30 breaths per minute and tachycardic with a heart rate of 145 beats per minute. He was febrile with a temperature of $39.0^{\circ} \mathrm{C}$. Oxygen saturation was $98 \%$ on room air. His left thumb was inflamed but there were no signs of any embedded bee body parts after removal by his wife. The remainder of his physical examination was unremarkable.

Investigations revealed a normal white cell count $10.5 \times 10^{9} / \mathrm{L}$ [reference range (RR) 3.50-11.00] with a mild neutrophilia of $8.29 \times 10^{9} / \mathrm{L}$ (RR $\left.1.7-7.0\right)$. Arterial whole blood showed a normal lactate $1.4 \mathrm{mmol} / \mathrm{L}$ (RR 0.0-2.2). He had normal renal and liver function test results and his urinalysis, electrocardiogram and chest X-ray were all unremarkable. One set of blood cultures was collected into BD BACTEC Plus Aerobic/F and Plus Anaerobic/F culture vials (Becton Dickinson, USA). They were incubated in the BD BACTEC 9000 automated blood culture system. After 4 hours observation and conservative management in the emergency department (ED), he was discharged home. His aerobic blood culture vial signalled positive 48 hours later with Gram-negative bacilli and he was recalled to the ED for further assessment. Clinically, he was doing well with no fever, thus he was not started on any antibiotic and was discharged home again.

The contents of the aerobic vial were subcultured onto horse blood, chocolate and MacConkey agars and these media were incubated at $35^{\circ} \mathrm{C}$. Growth was evident only on horse blood agar and chocolate agar incubated in $5 \% \mathrm{CO}_{2}$ after 48 hours incubation. There was no growth on horse blood agar incubated anaerobically and no growth on
MacConkey agar incubated in ambient air. Colonies were mucoid, grey-white, round, and convex with entire edges. Cells were non-motile, non-spore forming, long rods, occasionally filamentous in young cultures.

The blood culture isolate was further identified by matrixassisted laser desorption ionisation time-of-flight mass spectrometry (MALDI-TOF MS) using a Bruker MALDI Biotyper with Bruker Biotyper 3.4 software and library version V4.0.0.1 (Bruker Daltonik, Germany), according to the manufacturer's instructions, with a spectral score of 1.78 as Arsenophonus nasoniae with good confidence to the genus level.

Confirmation of the species identification was achieved by 16S rRNA PCR and sequencing. DNA extraction was performed from cultures by crude lysate preparation $\left(100^{\circ} \mathrm{C}\right.$ for 10 minutes in molecular grade water). The sequence obtained from the sample was $98.9 \%$ identical to the Arsenophonus nasoniae (ATCC 49151), which was deposited in GenBank (NCBI) under accession NR_042811.1, with eight mismatches in 796 bases sequenced.

Antimicrobial susceptibility testing of the A. nasoniae blood culture isolate was performed using the Vitek 2 semiautomated system (version 08.01; bioMerieux, USA) with the commercially available Vitek 2 AST-N246 card. Antimicrobial susceptibility results are shown in Table 1 .

The patient decided to keep the dead bee in a resealable plastic bag and its remnants were sent to Hawkesbury Institute for the Environment, Western Sydney University for further identification and possible detection of the causative bacterial DNA, 6 weeks after the contact.

The bee was morphologically confirmed as Apis mellifera. Culture was attempted on the bee remnant, but we were not able to identify $A$. nasoniae from the mixed growth. The remnant was homogenised in a TissueLyser (Qiagen, Germany) at $30 \mathrm{~Hz}$ for $4 \mathrm{~min}$ with a $2 \mathrm{~mm}$ sterile steel ball. Total DNA was then extracted using TRIsure (Bioline) following the manufacturer's recommendations. To detect the presence of Arsenophonus sp. DNA in the bee sample, PCR was performed using the Bioline MyTaq Red PCR kit and primer set Ars23S1 (CGTTTGATGAATTCATAGTCAAA)/

Ars23S2 (GGTCCTCCAGTTAGTGTTACCCAAC). ${ }^{2} 200$ ng DNA was used in a $10 \mu \mathrm{L}$ reaction $(2 \mu \mathrm{L}$ DNA, $2 \mu \mathrm{L}$ MyTaq

Table 1 Antimicrobial susceptibility of the Arsenophonus nasoniae blood culture isolate using VITEK 2 Systems Version: 08.01 and AST-N246 card

\begin{tabular}{lc} 
Antimicrobial agent & MIC (mg/L) \\
\hline Ampicillin & 4 \\
Amoxicillin/clavulanic acid & $\leq 2$ \\
Cefazolin & $\leq 4$ \\
Ceftriaxone & $\leq 1$ \\
Ceftazidime & $\leq 1$ \\
Cefepime & $\leq 1$ \\
Meropenem & $\leq 0.25$ \\
Amikacin & 4 \\
Gentamicin & $\leq 1$ \\
Tobramycin & $\leq 1$ \\
Ciprofloxacin & $\leq 0.25$ \\
Norfloxacin & $\leq 0.5$ \\
Nitrofurantoin & $\leq 16$ \\
Trimethoprim & $\leq 0.5$ \\
Trimethoprim/sulfamethoxazole & $\leq 20$ \\
\end{tabular}

MIC, minimal inhibitory concentration. 